
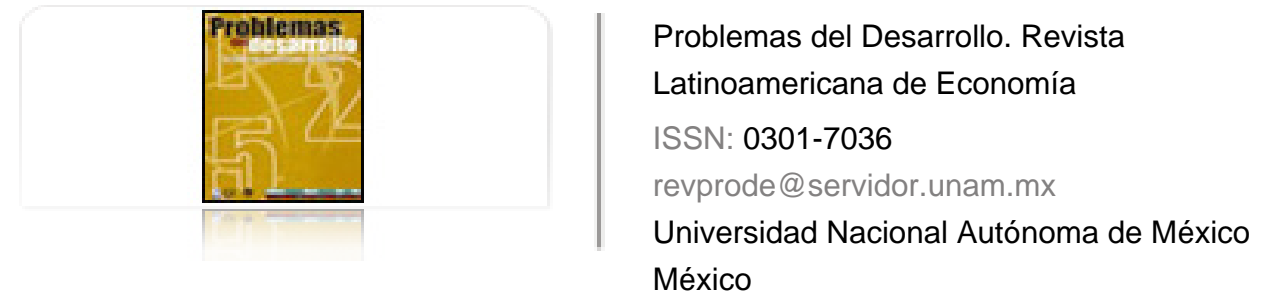

León de los Santos, Gabriel

Políticas públicas para la promoción de la cogeneración eléctrica en México Problemas del Desarrollo. Revista Latinoamericana de Economía, vol. 35, núm. 137, 2004, pp. 117-

133

Universidad Nacional Autónoma de México

Distrito Federal, México

Disponible en: http://www.redalyc.org/articulo.oa?id=11825947008

- Cómo citar el artículo

- Número completo

- Más información del artículo

Página de la revista en redalyc.org

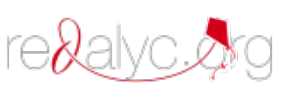

Sistema de Información Científica

Red de Revistas Científicas de América Latina, el Caribe, España y Portugal Proyecto académico sin fines de lucro, desarrollado bajo la iniciativa de acceso abierto 


\title{
PoLítICAS PÚBLICAS PARA LA PROMOCIÓN DE LA COGENERACIÓN ELÉCTRICA EN MÉXICO
}

\author{
Gabriel León de los Santos*
}

Fecha de recepción: 12 de marzo de 2004. Fecha de aceptación: 14 de junio de 2004.

\section{Resúmenes}

Se ha buscado el desarrollo de la cogeneración en el mundo mediante la promoción en los diferentes sectores con capacidad para implantarla. Esta labor fue impulsada desde los gobiernos y apoyada por organizaciones públicas gubernamentales y académicas, además de asociaciones privadas comerciales y fabricantes de equipos. Ello con la finalidad de dinamizar este proceso, aprovechar los potenciales, desarrollar este mercado, ampliar y robustecer el sector eléctrico, utilizar mejor la energía, mitigar los efectos contaminantes e intensificar el impulso a tecnologías más eficientes. En el caso de México ha sido menos exitoso, a juzgar por los resultados obtenidos desde 1992 hasta la fecha.

Palabras clave: cogeneración, CHP, potencial, promoción, desarrollo, obstáculos.

\section{Abatract}

The development of co-generation worldwide has been sought through its promotion within the different sectors that have the potential (i.e. the capacity to implement it) labor stimulated by governments and supported by public governmental and academic organizations, as well as private commercial associations and directly by the makers of the equipment. This has aimed to make the process more dynamic, achieving good use of the potential, developing the market, expanding and strengthening the electricity sector, making the best use of energy, mitigating any pollutive effects, and stimulating more efficient technologies. In the specific case of Mexico, this effort has been much less successful, given the results obtained since 1992.

Key terms: co-generation, CHP, potential, promotion, development, obstacles.

* Investigador del Departamento de Sistemas Energéticos, División de Ingeniería Eléctrica, UNAM. Correo electrónico: tesgleon@aol.com 


\section{Résumé}

On a cherché le développement de la cogénération dans le monde à travers sa promotion dans les différents secteurs à potentialité (capacité pour produire), tâche encouragée par les gouvernements et soutenue par les organisations publiques gouvernementales et académiques, en plus des associations privées commerciales et, directement, par les fabricants d'équipements. Ceci, afin que ce processus soit dynamique, afin qu'on réussisse à tirer profit des potentiels, qu'on développe ce marché, qu'on étende et qu'on fortifie le secteur électrique, qu'on réussisse une meilleure utilisation de l'énergie, qu'on mitige les effets polluants et qu'on encourage l'élan des technologies plus efficientes. Dans le cas du Mexique en particulier, elle a eu moins de succès, étant donné les résultats obtenus depuis 1992 et jusqu'à ce jour.

Mots clés: cogénéation, CHP, potentiel, promotion, développement, obstacles.

\section{Resumo}

O desenvolvimento da co-geração no mundo foi promovido nos diferentes setores com potencialidade e capacidade para implementá-la. Este trabalho foi impulsionado pelos governos e apoiado por organizações públicas governamentais e acadêmicas, e além disso, obteve o respaldo das associações privadas comerciais e interessou diretamente aos fabricantes de equipamentos. Esses esforços conjuntos visam tornar este processo mais dinâmico e aproveitar melhor os potenciais envolvidos. O objetivo é assegurar um desenvolvimento contínuo deste mercado, ampliando e fortalecendo o setor elétrico, com o uso adequado da energia, diminuição dos efeitos contaminantes e fomento de tecnologias mais eficientes. No caso do México, de acordo com os resultados obtidos de 1992 até a presente data, o desenvolvimento deste processo tem sido de pouco êxito.

Palavras-chave: co-geração, CHP, potencial, promoção, desenvolvimento, obstáculos. 


\section{Introducción}

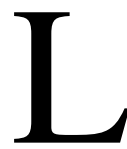

a cogeneración es una técnica que permite producir dos o tres tipos de energía a partir de una fuente primaria, por ejemplo, electricidad, vapor y frío a partir de gas natural. Este uso más eficiente de la energía ofrece numerosas ventajas desde diversos puntos de vista.

En el plano económico, permite una reducción global de hasta $30 \%$ en el costo de producción. ${ }^{1}$ De igual modo, aumenta la disponibilidad y confiabilidad del abasto energético en la industria. En el plano ambiental mitiga las emisiones contaminantes a la atmósfera. Además, ${ }^{2}$ disminuye la demanda de electricidad al servicio público. Con esto, la empresa eléctrica se libera de dicha demanda y puede destinar esa capacidad a otro consumidor. Como objetivo central, la cogeneración busca el autoabastecimiento de energía, no su venta. Con base en este propósito, estudios de la OCDE distinguen tres tipos de esta técnica: industrial, a gran escala (petroquímicas, industrias grandes) y a pequeña escala (micro cogeneración). Asimismo denominan potencial de cogeneración técnico al que no requiere combustible adicional (también denominado ${ }^{3}$ potencial natural). Así, pueden diferenciarse dos clases de potenciales y, derivados de ellos, dos tipos de excedentes eléctricos: los naturales, que no requieren combustible adicional, y los comerciales, que utilizan combustible adicional y del nivel que se deseen (no tienen límite o potencial definido, sino que se fijan por el nivel de combustible que se elija usar, en función de los fines o interés del dueño del proceso industrial que utiliza el esquema de cogeneración o del interés del concesionario del permiso). Esta diferenciación ${ }^{4}$ no la consideran la reglamentación mexicana ni los estudios del potencial nacional de cogeneración que realizó la CONAE en 1995.

\footnotetext{
La diferencia de eficiencia convencional media global (para el caso de México, de 56.7\%), contra la media del esquema de cogeneración máxima ( $86 \%)$, muestra potenciales de ahorro económico de hasta $30 \%$ en los costos de suministro y abasto del insumo energético. Este es un dato técnico que los fabricantes y desarrolladores utilizan como elemento de promoción comercial para la venta de sus equipos.

2 Estos beneficios se refieren a su condición de potencial. El nivel particular de beneficio del esquema de cogeneración que se quiera implantar ubicará sus beneficios económicos en este rango ( 0 a $30 \%$ ), y dependiendo del nivel que alcance y de las condiciones de financiamiento, será o menos viable que otros esquemas y potenciales particulares. Esto en términos de la evaluación financiera que se realice (VPN, TIRM, RBE, entre otros).

3 Estudio realizado para la tesis doctoral: "Estudio de la viabilidad de la cogeneración industrial en México", G. León, DEPFI, UNAM, 2003.

4 Se estima el potencial industrial de cogeneración considerando este concepto. Esto es, un arreglo de cogeneración tiene un nivel definido de excedentes de calor y electricidad (técnicos o naturales), dados por el nivel de recuperación de energía de rechazo del proceso. Y si se quiere, se puede
} 
Este trabajo ${ }^{5}$ muestra los resultados ${ }^{6}$ del desarrollo de la cogeneración en México hasta 2002, sus perspectivas de crecimiento en comparación con algunas experiencias en otros países y zonas. Además, se plantean algunos argumentos para explicar los resultados vistos. ${ }^{7}$ Se revisan los aspectos más importantes de las promociones, uno de los cuatro aspectos más relevantes del marketing, ${ }^{8}$ basados en la estrategia de promoción comúnmente usada para la cogeneración, con el fin de identificar sus aspectos fundamentales y resulta$\operatorname{dos}^{9}$ en México y en otras regiones con experiencias exitosas.

\section{Avance de la cogeneración en otros países}

Por sus amplias ventajas, la cogeneración ha despertado el interés de empresas y gobiernos, lo que ha llevado a su rápido desarrollo en muchos países.

En Estados Unidos, y con el fomento de la Public Utility Regulatory Policy Act (PURPA) de 1978, en 2000 había en operación 65900 mwe. ${ }^{10}$ En España, desde la promulgación de la Ley de conservación de energía en 1980, se logró aumentar de 694 a 5,912 mwe en 1999.

En Italia, los mwe aumentaron en 1994 de 5 660, a 10482 en 2001. Cabe hacer mención de que en Europa los niveles más altos de aprovechamiento de la cogeneración se presentan en Dinamarca, con 35\%. En el resto del mundo, los países con mayor capacidad instalada son Estados Unidos y China (27 712 mwe).

aumentar el nivel de excedentes con equipos de combustión adicionales; pero estos excedentes ya no pueden denominarse técnicos o naturales, ya que no provienen de un proceso de recuperación y optimización en el uso de la energía, sino de un proceso de generación de vapor con combustible adicional al de la cogeneración, para su uso en diferentes aplicaciones, como generación eléctrica, para abasto de un proceso industrial o su comercialización.

5 El tema del estado y papel de la promoción de la cogeneración sólo plantea mostrar el desarrollo y estado que ésta tiene en México (de 1992 a 2003), y su perspectiva en términos de sus potenciales.

6 En otros trabajos se analizan los elementos que promueven y obstaculizan el desarrollo de la cogeneración en México. Una de las conclusiones es que en el periodo 1992 a 2000 su desarrollo ha sido demasiado lento. En este trabajo no se discute este tema.

7 El precio y disponibilidad del gas natural comenzaron a ser factores clave para el desarrollo de la cogeneración a partir del año 2000. Los resultados vistos hasta ahora son producto de las condiciones existentes de 1992 a febrero de 1999. Los efectos del precio del gas y su disponibilidad comenzarán a reflejarse a partir de 2004, ya que la maduración e implantación de los proyectos de cogeneración tardan entre tres y cuatro años. Pero en esos resultados tendrá mucho mayor impacto la incertidum bre generada por el intento de reforma del sector eléctrico y las nuevas reglas, ya que no es conside rada ni mencionada.

Este trabajo no pretende explicar el porqué de las condiciones, sino mostrar los resultados obtenidos y cuáles han sido las estrategias para promocionar su desarrollo en México y en otros lugares, comparando los resultados vistos.

8 Los cuatro pasos del marketing son: precio, producto, plaza y promoción. En este trabajo se analizan las herramientas de mayor importancia, los objetivos y las estrategias de promoción.

9 Que consiste en vender al usuario final la idea del desarrollo de la cogeneración.

10 $\quad$ MWe $=$ megawatts eléctricos. 


\section{Situación de la cogeneración en México}

En contraste con el rápido crecimiento de la cogeneración en muchos países, en México ha sido relativamente lento. Desde el primer intento de desarrollo a finales de los años setenta y pasando por la reforma de 1992, se estima ${ }^{11}$ que en 2002 operaban 1,132.3 mwe en la industria privada.

Sin embargo, desde que se modificó la Ley del servicio público de energía eléctrica en 1992 y se abrió el mercado eléctrico a la participación de los particulares en las modalidades de productor independiente, autoabastecimiento, cogeneración, importación y exportación, ha crecido a un ritmo promedio anual muy bajo, con una participación en la capacidad nacional de $0.54 \%$ en 1995 y $2.74 \%$ en $2002 .{ }^{12}$

Si la cogeneración ofrece a la industria beneficios de ahorro en la producción eléctrica, a la compañía eléctrica en inversión en infraestructura, y al país en el consumo de energéticos y emisiones contaminantes, ¿por qué han dado pobres resultados en México?, ¿será que el potencial no es tan importante?, ¿sus costos en el país la hacen poco competitiva frente a la opción de abastecimiento mediante el servicio público?, ¿acaso los factores institucionales son el principal obstáculo?, ¿son los empresarios quienes no ven satisfechas sus expectativas para el desarrollo de su potencial?

Para responder a estas preguntas debemos considerar los puntos de vista de los diferentes actores involucrados desarrolladores o fabricantes de equipos, suministradores de combustibles, usuario final, empresa pública del servicio eléctrico, ente regulador, gobierno, organismos empresariales, sector académico y organismos promotores de su desarrollo. El debate es complejo, ${ }^{13}$ y en el fondo debiera regirse por lo que se quiera hacer con esta técnica en el ámbito nacional (empresas públicas, preservación del recurso energético y del medio ambiente) y en el privado (trasladar segmentos de mercados del proveedor público a privados, abrir nuevas oportunidades de negocios particulares a expensas de las empresas públicas, o como lo mencionan los argumentos oficiales, dar mayor competitividad a estos sectores, modernizándolos y aliviando la carga financiera del gobierno al trasladar la obligación de invertir a los particulares).

11 Comisión Reguladora de Energía (CRE a 2002), con base en permisos otorgados y cuyo estatus está en operación. Pero también reporta $2798.6 \mathrm{MW}$ por autoabastecimiento, así que los datos no son claros respecto a cuánto del autoabastecimiento es por cogeneración y cuánto convencional.

12 Estimación propia con base en los permisos otorgados por la CRE para la modalidad de cogeneración otorgados en 1995, y 2.74\% con datos del 2002.

13 El nivel de aprovechamiento de ese potencial depende de la viabilidad financiera del proyecto de cogeneración. Un elemento importante lo constituye el precio del combustible, pero mucho más el precio y cantidad de excedentes eléctricos que se puedan vender. El incremento el costo del gas mediante un análisis incremental entre costos convencionales y por cogeneración, puede cancelar una parte importante del incremento del costo debido a los precios, pero no la incertidumbre de su disponibilidad (aunque puede reducirse considerando equipo dual). 
La respuesta más generalizada es la que relaciona su éxito o fracaso con la rentabilidad económica financiera, argumento, sin duda, inobjetable. Al contrario de Estados Unidos, España, e Italia, en donde se reconoce el costo por capacidad y no sólo el del combustible en el precio de compra de los excedentes eléctricos en México no se han generado incentivos para su desarrollo. Esto se debe seguramente a problemas de orden político y refleja la visión dominante en la reglamentación. ${ }^{14}$

\section{Por qué promover la cogeneración}

La importancia que le conceden algunos países a esta técnica queda demostrada en la formación de asociaciones y grupos de trabajo para su desarrollo y promoción. Un ejemplo es el Grupo Europeo de Cogeneración (GEC), cuyo objeto es realizar tareas de promoción en la Unión Europea (UE). Sus miembros incluyen más de 190 compañías de electricidad y autoridades de 30 países. Su actividad prioritaria es investigar el nivel de desarrollo en la ue para dar a conocer su situación a esa comunidad.

Estas organizaciones explican los beneficios, reúnen información sobre la situación actual de la cogeneración en las industrias eléctricas locales para un mercado estratégico y, difunden la información a todos los miembros mediante publicaciones especializadas. El GEC se ha concentrado en cuatro temas para posicionar la cogeneración en un lugar trascendente en el desarrollo sustentable de Europa: $a$ ) ambiental, b) económico, $c$ ) político, y d) comercial.

El potencial en la Unión Europea (véase en la Tabla 1) representa grandes montos de inversión, así como la oportunidad de preservar los recursos energéticos, mitigar las emisiones de gases y desarrollar tecnologías y comercializarlas dentro y fuera de su región. En la Tabla 2 se muestran las estimaciones que sobre estos rubros se tienen para el año 2010.

14 Ello se debe fundamentalmente a que el marco legal dentro del cual se planeó el desarrollo del autoabastecimiento, ya sea por cogeneración o por otros métodos, presenta las siguientes limitaciones:

- La aparición con casi seis años de retraso respecto a la apertura del mercado eléctrico de la reglamentación para el respaldo eléctrico y el acceso a la transmisión.

- La imposición del despacho, que sujeta el nivel de producción industrial a la demanda eléctrica.

- Falta de certidumbre para la determinación del precio de compra de los excedentes eléctricos (tomados a 85 y 90\% del costo marginal regional (CMH) incurrido en el periodo horario (valor del nodo más cercano de entrega, dado por la energía más económica que entrega y genera CFE en dicho nodo, información sólo conocida por CFE), y por el precio subastado (PS). Bajo la moda lidad que aplica a la generación por cogeneración incisos b), c) y d) Cargo por la energía entre gada. Véanse las relaciones C41,C42,C43 sobre cargos por servicios de transmisión).

- Topes en la cantidad de energía entregada a la red (20Mw).

- Vacío en el manejo para los excedentes térmicos. 
Tabla 1

Potencial de cogeneración técnico para 2020 en países europeos (Mwe)

\begin{tabular}{lccr}
\hline \multicolumn{1}{c}{ País } & Industrial & Pequeña y gran escala & Total \\
\hline Alemania & 7876.7 & 34474.9 & 42351.6 \\
Francia & 4223.7 & 6392.7 & 10616.4 \\
Inglaterra & 3653.0 & 10958.9 & 14611.9 \\
Italia & 3310.5 & 1141.6 & 4452.1 \\
Holanda & 2397.3 & 4794.5 & 7191.8 \\
Suecia & 2397.3 & 4452.1 & 6849.3 \\
España & 2168.9 & - & 2168.9 \\
Finlandia & 1712.3 & 1369.9 & 3082.2 \\
Bélgica & 1027.4 & 3310.5 & 4109.6 \\
Austria & 684.9 & - & 3995.4 \\
Portugal & 570.8 & 1826.5 & 2168.9 \\
Dinamarca & 342.5 & 342.5 & 570.8 \\
Irlanda & 228.3 & - & 228.3 \\
Grecia & 228.3 & 114.2 & 228.3 \\
Luxemburgo & 114.2 & 72260.3 & 103196.3 \\
\hline Total uE & 30936.1 & &
\end{tabular}

Fuente: elaboración propia con datos de Projected Costs of Generating Electricity Update 1998, OCDE.

Tabla 2

Potencialidad técnica, económica y comercial de la cogeneración en la Unión Europea

\begin{tabular}{ll}
\hline \multicolumn{1}{c}{ Actividad } & \multicolumn{1}{c}{ Estimación } \\
\hline Participación media en la generación total & $10 \%$ \\
Participación media esperada para 2010 & $18 \%$ \\
Capacidad instalada & $65-70000 \mathrm{Mwe}$ \\
Capacidad instalada esperada para 2010 & $130000 \mathrm{Mwe}$ \\
Capacidad adicional & $60-65000 \mathrm{Mwe}$ \\
Inversión adicional & $50-60$ billones de euros \\
Inversión anual media esperada en cogeneración & 5 billones de euros por año \\
\hline
\end{tabular}

Fuente: Who's Who in European Cogeneration 2000-2001. COGEN Europe, septiembre de 2000.

Por otra parte, Estados Unidos, mediante el U.S. Department of Energy y la Office of Distributed Energy and Electric Reliability, promueve ${ }^{15}$ un programa que busca alcanzar los 92 Gwe en 2010 por medio de:

1. El incremento de la difusión de la cogeneración, iniciativas regionales, congresos, seminarios y actividades educativas y de promoción.

15 CHP Partners' Meeting, Washintong, DC, abril, 2003. 
2. La eliminación de las barreras regulatorias, institucionales y técnicas; análisis y recomendaciones sobre prácticas de interconexión y tarifas, costos de instalación, opciones de financiamiento y lecciones aprendidas; clasificación de estructuras eléctricas; normatividad del cuidado del aire; escenarios de precios; estrategias federales impositivas para fomentar la adopción de la cogeneración; incentivos fiscales para la adopción de tecnologías para el uso eficiente de la energía.

3. El desarrollo de mercados y tecnologías de la cogeneración (CHP), junto con el análisis de las oportunidades para acceder a nichos de mercado para la cogeneración; estimación de la capacidad instalada de cogeneración.

4. El análisis de la generación distribuida (hoja de cálculo para medir los beneficios de la cogeneración en emisiones evitadas, selección de herramientas preliminares).

Para dar apoyo a esta iniciativa se cuenta con la asistencia del Midwest Regional cHP Applications Center, de la Universidad de Illinois en Chicago.

Asimismo, la Climate Protection Partnerships Division de la Environmental Protection Agency y Combined Heat and Power Partnership promueven el desarrollo del mercado de la cogeneración identificando segmentos específicos en diversas zonas de Estados Unidos. Éstos trabajan en equipo con ERG Inc., D\&R International, los estados del medio este y noroeste, el Departamento de Energía y socios comprometidos; dando asistencia técnica, además de difundir las experiencias y estimar e identificar los potenciales por sectores y segmentos de mercado como plantas de etanol, productos lácteos y hospitales.

Tabla 3

Potencial de cogeneración para 2010 en Estados Unidos

\begin{tabular}{lllr}
\multicolumn{1}{c}{ Industrial } & $G W$ & Servicios & $G W$ \\
\hline Petroquímica & 9 & Oficinas & 10 \\
Siderurgia & 6 & Hospitales & 7 \\
Manufacturas & 4 & Universidades & 7 \\
Celulosa y Papel & 3 & Escuelas & 3 \\
Procesadoras y empacadoras & & & \\
$\quad$ de alimentos & 2.3 & Hoteles & 1.8 \\
Plásticos & 1.9 & Restaurantes & 1 \\
& & Apartamentos & 1 \\
& & Supermercados & 0.8 \\
\hline Total & 26.2 & & 31.6 \\
\hline
\end{tabular}

Fuente: elaboración propia, datos de Resource Dynamics, mayo de 2002.

Los objetivos específicos o metas cuantitativas de este nuevo impulso al desarrollo de la cogeneración en Estados Unidos (véase Tabla 4) aprovechar 45\% del potencial disponible, lo que representa un mercado de 15600 mdd en venta de servicios y equipo. 
Tabla 4

Meta de aprovechamiento

al año 2010 en Estados Unidos (GWe)

\begin{tabular}{lcc}
\hline & Potencial & Meta \\
\hline Servicios & 31.6 & 14.3 \\
Industrial & 26.2 & 11.8 \\
\hline Total & 57.8 & 26.1 \\
\hline
\end{tabular}

Fuente: elaboración propia, datos de Resource Dynamics, mayo de 2002.

Para el caso de México, ${ }^{16}$ se muestra en la Tabla 5 que, con un crecimiento moderado, su potencial ${ }^{17}$ podría ser muy importante en comparación con los que están por desarrollarse en los países europeos.

Tabla 5

Potencial de cogeneración técnico ${ }^{18}$ al año 2020 en México (mwe)

\begin{tabular}{ccc}
\hline Industrial & pequeña y gran escala & Total \\
\hline 32590 & 4000 & 36590 \\
\hline
\end{tabular}

Fuente: elaboración propia. ${ }^{19}$

En México las expectativas podrían ser similares, sólo que las inversiones representarían erogaciones importantes ${ }^{20}$ que saldrían del país (18,917 mdd en 20 años), ya que de los montos de inversión, del equipo de cogeneración es el principal y en el país no se diseña y se fabrica muy poco. A esto debe añadirse la oportunidad de preservar los recur-

16 Idem.

17 Incluye el potencial de PEMEX.

18 Bajo un análisis de viabilidad económico y financiero.

19 Idem.; y 4,000 MWe estimados en PEMEX (José M. Muñoz, 2000), de los cuales existe intención de obligar a su aprovechamiento por parte del Poder Legislativo (...el artículo tercero transitorio dispone que en un plazo de ocho años la empresa paraestatal deberá contar con una capacidad instalada de cogeneración suficiente para producir, cuando menos, 4 mil MW, y señala que estos proyectos deberán iniciarse en los estados de la república de menor desarrollo económico).

La CONAE en su estudio para el potencial de cogeneración del año 1995, determinó un valor de entre 7500 y 14000 Mw. El potencial que se menciona en el artículo estima que para 2016 este podrá ser de 32590 MW (valor, metodología y consideraciones de cálculo verificable en el trabajo referenciado, en la misma tabla).

El crecimiento promedio estimado para México puede ubicarse en 5\% anual (asumiendo que el industrial sufra el mismo comportamiento), entonces proyectando con base en esto:

$$
14000(1+.05) 21=39003 \mathrm{MW}
$$

Con lo cual se obtiene un potencial de cogeneración para 2016 con base en el cálculo previo o base, hecho por la CONAE. Entonces, $32560 \mathrm{MW}$ es un valor razonable.

20 Con base en el costo medio de $0.517 \mathrm{mdd} / \mathrm{MWe}$ calculado con los datos reportados por la CRE. 
sos energéticos, mitigar las emisiones e iniciar el desarrollo de tecnologías propias y comercializarlas dentro y fuera del país.

\section{Promoción de la cogeneración en México}

En México, dada la importancia ${ }^{21}$ que se le ha atribuido a la cogeneración en los ámbitos legislativo, gubernamental, comercial y académico, se pueden identificar tres tendencias o etapas de desarrollo de la cogeneración, con base en la importancia e impulso que desde el gobierno se le ha dado:

1) En el periodo 1990-1995, una política basada sólo en dos de los cuatro fundamentos que propiciaron este fenómeno: ${ }^{22} a$ ) reducción de los impactos ambientales, $b$ ) aprovechamiento de potenciales eléctricos basándose en energías renovables, $c$ ) técnicas de

21 En los años ochenta la industria eléctrica buscó la independencia de los recursos energéticos externos, menos volatilidad de los precios al público y la reducción de las emisiones de gases de invernadero. Para ello se promovió el uso de combustibles más limpios como el gas natural, el desarrollo de los potenciales eléctricos basados en las energías renovables y técnicas de generación más eficientes, como los ciclos combinados y la cogeneración.

Después, a principios de los noventa, este primer impulso fue sustituido por la visión del neoliberalismo, la cual ya no considera útil la integración vertical de las industrias eléctricas, con lo que el modelo dejó de ser la base de la estructura de las éstas en el mundo (la desintegración vertical de las empresas eléctricas en muchos países así lo muestra). A partir del proceso iniciado en Estados Unidos, el fundamento expresado es el de imprimir en la operación de la industria, más competitividad, apertura de mercados y reducción de tarifas, mediante la privatización y segmentación de los servicios eléctricos.

22 Durante décadas, las empresas eléctricas en Estados Unidos sufrieron incrementos en su demanda y decremento en sus precios. La economía de escala se mantuvo gracias a la capacidad adicional, los avances tecnológicos y la reducción de costos. Esta situación prevaleció hasta los años sesenta, cuando las empresas vieron disminuir sus gastos y crecieron rápidamente. Una serie de sucesos propicó un gran desarrollo de la industria eléctrica a finales de los sesenta:

- En 1965, el apagón del noroeste llamó la atención sobre la estabilidad del sistema eléctrico.

- La promulgación del Acta del Aire Limpio en 1970 y su reforma en 1977, la cual exigía a compañías eléctricas disminuir sus emisiones contaminantes.

- El embargo petrolero árabe en 1973 1974, que resultó en un incremento de los precios de los combustibles de origen fósil.

- El accidente, en 1979, en la isla Tres Millas, que produjo grandes costos y leyes reguladoras, además de una gran incertidumbre en el desarrollo de las plantas de energía nucleo eléctrica.

- Gran inflación en los precios de los energéticos causada por estos sucesos.

Mientras la industria se recuperaba de estos sucesos, el congreso preparaba una legislación para reducir la dependencia de los Estados Unidos de los combustibles fósiles mediante el desarrollo de energías renovables y alternativas más económicas y el uso más eficiente de los combustibles fósiles. El resultado de estos hechos fue la Public Utility Regulatory Policy Act de 1978 (PURPA). Ésta buscaba introducir mecanismos y facilidades para fomentar la competencia, así como los criterios de eficiencia impulsados por la FERC para ingresar a un mercado competitivo.

Inicialmente, las compañías eléctricas no aceptaron estas reformas, pero algunos encontraron ventajas, en especial, la disminución en la incertidumbre de la recuperación de los costos de inversión. El crecimiento de la capacidad de generación con la introducción de nuevas formas de compañías 
generación más eficientes, y $d$ ) reducción de la dependencia energética del exterior. Situación manifiesta por las reformas de 1991 y 1992, y promovida con intensidad hasta 1995 con resultados muy pobres en cuanto al desarrollo de los potenciales existentes en el país.

2) Para el periodo 1996-1999 se descarta como política de desarrollo o apoyo al sector eléctrico nacional el impulso de energías renovables y técnicas de generación más eficientes, quedando a nivel de medidas de ahorro de energía. Y con esto se pasa a la segunda etapa de las tendencias internacionales,

3) Y a partir de 1999, y durante la actual administración, la desintegración vertical y la privatización de la industria eléctrica, que se refleja en la reducción en el impulso a las energías renovables, la política de apertura y privatización de los mercados del gas natural.

Por otra parte, diversos organismos institucionales se han dedicado a analizar la problemática de la cogeneración en México y a promover su entendimiento, difusión y desarrollo en una forma más rápida y eficiente.

La $\operatorname{CONAE}^{23}$ aborda el tema desde el punto de vista de la promoción y asesoría técnica a las industrias. Dentro de su Programa de Desarrollo y Reestructuración del Sector de la Energía, sus estrategias sectoriales consideran: $a$ ) concertar con los sectores social y privado su incorporación a los esfuerzos nacionales de ahorro, $b$ ) continuar el desarrollo de la capacidad humana, organizacional e institucional, $c$ ) participar en el desarrollo del marco regulatorio en materia de eficiencia energética para adecuarlo y complementarlo con el actual.

Para lo cual hace uso de sus funciones, que se dividen en: 1) normalización, 2) asistencia técnica y 3) promoción. Y dentro de ésta: a) concebir y promover estrategias y acciones, así como coordinar y concertar dichas acciones, $b$ ) preparar, coordinar y evaluar los programas nacionales de ahorro de energía, $c$ ) promover, fomentar y difundir estudios relacionados con la utilización de energía, así como la investigación, desarrollo y difusión de tecnología para el ahorro y uso racional y eficiente de la energía. El trabajo de promoción de la cogeneración se basa en:

- evaluación del potencial nacional de cogeneración

- evaluación de proyectos específicos

- elaboración de manuales

- promoción de proyectos

eléctricas era un adelanto contenido en el Acta Política de Energía de 1992 (ЕРACT). ЕРACT expandió el mercado eléctrico con productores de este sector.

23 Odón de Buen Rodríguez, "Estrategias de promoción de la cogeneración y generación de energía eléc trica a partir de energías renovables", Comisión Nacional para el Ahorro de Energía, México, 1997. 
- seminarios de cogeneración

- capacitación de recursos humanos

- el trabajo de la Subcomisión de Promoción de Proyectos de Cogeneración (SPPC)

Su estrategia de fomento y desarrollo se fundamenta en identificación de barreras, de fuentes de financiamiento y promoción de esquemas específicos, asistencia técnica, difusión de los beneficios, y establecimiento de espacios y mecanismos de acción concertada de los diversos agentes interesados. Esto se comprueba al revisar sus principales trabajos durante el periodo 1992-2003.

Una de sus principales actividades es la realización de congresos internacionales en cuanto a cogeneración y energías renovables se refiere, que desde 2002-2003 han sumado nueve. También la CONAE, con apoyo del Instituto para la Diversificación de la Energía de España realizó en 1992 una estimación del potencial de cogeneración del país hasta 1998. Además, en 1996 se llevó a cabo un cálculo similar en 1995. Se creó la SPPC, cuyos objetivos son analizar los obstáculos que impiden o limitan el desarrollo de la cogeneración en México, definir estrategias para eliminar dichos obstáculos y promover proyectos específicos identificados por su viabilidad técnica y económica. En 1997 (Nieva et al., 1996) se publicó el estudio que realizó el Instituto de Investigaciones Eléctricas (IIE), ${ }^{24}$ a encargo de la CONAE, acerca del potencial nacional de cogeneración como opción en la expansión del sistema eléctrico nacional, con base en el potencial que la CONAE determinó para 1995.

De acuerdo con su plan de trabajo CONAE 2001-2006, ${ }^{25}$ y en línea con la política de la administración del presidente Fox — la cual definió nuevas reglas para integrar capacidad de generación de electricidad a la Red Eléctrica Nacional por parte del sector privado-, la CONAE concentró sus actividades en apoyar el diseño de políticas de fomento y promoción de la cogeneración en nuestro país mediante las siguientes líneas de acción:

- Integrar, con la participación de los principales interesados, propuestas de política pública para eliminar las barreras existentes relacionadas con la cogeneración.

- Promover la vinculación entre las diversas instituciones, públicas y privadas, nacionales e internacionales, interesadas o ralacionadas con el desarrollo del potencial de cogeneración en México.

- Continuar el desarrollo, integración y difusión de información relativa a la tecnología, oportunidades y marco regulador de la cogeneración en México.

24 Rolando Nieva, Julio A. Hernández, Enrique Portes y José M. Álvarez, Evaluación del potencial nacional de cogeneración como opción en la expansión del sistema eléctrico nacional, IIE, México, 1996. Aplicaciones tecnológicas, Boletín IIE, septiembre octubre 1997.

$25 \mathrm{http}: / / \mathrm{ww}$.conae.gob. $\mathrm{mx} / \mathrm{wb} /$ distribuidor.jsp?seccion $=411 \# \operatorname{cog}$ 
Para ello busca trabajar en coordinación con los actores clave para el éxito de su programa: secretarías de Energía, de Hacienda y Crédito Público, y Economía; Comisión Reguladora de Energía; CFE; LYFC, PEMEX; fabricantes y proveedores de sistemas de cogeneración, distribuidores de combustible, cámaras y asociaciones industriales, instituciones de financiamiento, grupos de consultoría y desarrolladores.

Su meta cuantitativa para el periodo 2001-2006 (Tabla 6) no es clara:

Tabla 6

Tres de las cinco metas rectoras establecidas para el periodo 2001-2006

\begin{tabular}{llc}
\hline \multicolumn{1}{c}{ Meta } & \multicolumn{1}{c}{2001} & 2006 \\
\hline $\begin{array}{l}\text { Impacto de los programas de gran alcance } \\
\text { de la conAE (ahorros) }\end{array}$ & Mw $=20$ & Mw = 300 \\
$\begin{array}{l}\text { Número de empresas apoyadas en ahorro } \\
\text { de energía y energía renovable (anual) }\end{array}$ & Asistencia técnica $=3000$ & Asistencia técnica $=10000$ \\
$\begin{array}{l}\text { Número de recursos humanos } \\
\text { capacitados en ahorro de energía } \\
\text { y energías renovables (anual) }\end{array}$ & $\begin{array}{l}\text { Número de } \\
\text { capacitados }=3700\end{array}$ & $\begin{array}{l}\text { Número de } \\
\text { capacitados }=6000\end{array}$ \\
\hline
\end{tabular}

Fuente: CONAE.

Por su parte, el FIDE capacita en ahorro de energía eléctrica, difusión, asistencia técnica, financiamiento y promoción de proyectos, y en 2004 desarrolla un proyecto de microcogeneración, dando financiamiento de hasta de $2.5 \mathrm{mdp}$. Sin embargo, este programa es promocionado por los desarrolladores y fabricantes de equipo, por lo que la tarea del FIDE en este ámbito es modesta, y más bien correspondería a los programas de financiamiento para la modernización tecnológica y uso eficiente de la energía.

Es establecimiento de la cogeneración, además de promocionarse, debe financiarse. En México se ofrecen varios tipos de apoyos financieros, ya que al tratarse de un cambio tecnológico para modernizar la planta industrial, optimizar el uso de combustibles, mejorar la productividad industrial, y combatir las emisiones contaminantes, puede impulsarse desde el campo financiero con programas de fomento y apoyo a las plantas industriales. Por tal motivo es fundamental la existencia y accesibilidad a estos tipos de programas. Pese a la intención de fomentar cambios y mejoras tecnológicas en México, el éxito de estos programas ha sido modesto. Entre ellos podemos mencionar:

- Nacional Financiera tiene como objetivos fundamentales coadyuvar a la preservación de la planta productiva e impulsar proyectos de inversión viables que estimulen la generación de empleo y el crecimiento económico, mediante:

- PROMIN: Programa Único de Financiamiento a la Modernización Industrial. 
- Banco Nacional de Comercio Exterior (BANCOMEXT).

- Por su parte, CONACYT tiene varios programas de financiamiento, como:

- FIDETEC. Fondo de Investigación y Desarrollo para la Modernización Tecnológica.

- ForCCYTEC. Fondo para el Fortalecimiento de las Capacidades Científicas y Tecnológicas Estratégicas.

- $\quad$ PMT. Programa de Modernización Tecnológica.

- En México se ofrecen varios tipos de financiamiento que pueden fomentar el desarrollo de la cogeneración:

- Proyectos de inversión (modernización energética o ampliación del proceso).

- Adquisición de bienes de capital (maquinaria y equipo).

- Asimismo, existen los siguientes programas para fortalecer el mercado activo de servicios tecnológicos y de mejoramiento ambiental relacionados con la cogeneración:

- Modernización tecnológica, que considera los siguientes aspectos:

- Evaluación tecnológica empresarial con el fin de dar prioridad a proyectos tecnológicos.

- Optimización de procesos.

- Reducción de costos de producción.

- Implicaciones de tendencias tecnológicas.

- Medidas de mejoramiento ambiental.

- Capacitación técnica sobre los temas mencionados.

- Mejoramiento ambiental, con objeto de controlar, mitigar o eliminar contaminación. Aquí sí existe una clasificación específica para la cogeneración:

- Proyectos de cogeneración y generación de energías renovables.

Todos estos programas ofrecen financiamiento, siempre y cuando la empresa genere divisas.

La tasa de interés de referencia es la TIIE para moneda nacional y LIBOR para la extranjera, más el margen que establezca el intermediario financiero. El plazo se determina en función de las necesidades del proyecto, hasta un máximo de 20 años, con el periodo de gracia que requiera. El monto máximo de los créditos en moneda nacional se calcula con base en el tamaño de la empresa y de ese modo se otorga para:

- La micro: hasta 1.7 millones.

- La pequeña: hasta 17 millones. 
- La mediana: hasta 40 millones.

- La grande: a partir de las características del proyecto.

Se puede ver que la promoción de la cogeneración depende básicamente de la CONAE, y que varios organismos que la financian, además de los desarrolladores y fabricantes de equipos y la banca comercial.

\section{Conclusión}

La promoción de la cogeneración busca fomentar el desarrollo de esta técnica. Esta labor la realizan dependencias gubernamentales, asociaciones privadas o una combinación de ambas, y en general las estrategias son las mismas: identificación de barreras regulatorias, institucionales y técnicas; análisis y recomendaciones sobre prácticas de interconexión, tarifas y respaldo; asesoría técnica y financiera; difusión de las experiencias, sugerencias para su adopción, incentivos impositivos; análisis de las oportunidades de acceso a segmentos de mercados; estimación de potenciales y de resultados de los programas de aprovechamiento; diseño y acceso a herramientas de evaluación de sus beneficios.

En España, esta promoción la realizó en primera etapa un solo organismo gubernamental (IDAE) y posteriormente, debido a su integración al bloque europeo, también el GEC como parte, de las tareas que realiza en la Unión Europea.

En Estados Unidos, la promoción es en el nivel gubernamental y mediante asociaciones, con una estrategia similar en los años setenta, ochenta, y en la campaña de principios del nuevo milenio.

En México, la promoción recae en un solo organismo gubernamental, la CONAE, la cual ha seguido una estrategia similar a la española. Sería recomendable ${ }^{26}$ que la SEMARNAP promocionara el desarrollo de la cogeneración, tal vez imitando la participación de la EPA en la experiencia de Estados Unidos, pero sólo ha incidido en la promoción de manera indirecta mediante su Programa Federal de Industrias Limpias. Por su parte, las organizaciones empresariales no desarrollan ninguna labor de promoción independiente y se reducen a complementar el trabajo realizado por la CONAE.

La promoción que se realiza en el país bien podría concentrarse en los factores clave para mejorar la percepción empresarial de las ventajas de la cogeneración:

26 Pero es claro que la SEMARNAP, y no sólo ella, sino las secretaría de Energía y de Economía siguen la po lítica indicada por el Ejecutivo Federal que no ha dado muestras de interés en el desarrollo de la cogeneración.

27 Esto es importante ya que la regulación en México no distingue ni hace mención de esto, y de aquí es de donde se han desprendido las imputaciones éticas y legales (solicitud de auditoría a la operación 
- Estabilidad en el marco regulador.

- Eliminación del tope de venta de excedentes.

- Mejoramiento en la metodología para determinar el precio de venta de los excedentes, distinguiendo ${ }^{27}$ entre los naturales y los comerciales.

- Seguridad en el abasto de los combustibles a precio de fomento para los excedentes naturales y a precio normal para los comerciales.

- Diligencia, prontitud y eficacia en la acción gubernamental.

Para garantizar cierto nivel de resultados, ${ }^{28}$ y que no suceda lo que ha venido ocurriendo durante los últimos diez años, será necesario que cambie la política gubernamental respecto de la cogeneración. El gobierno actual se preocupa por la privatización del sector eléctrico y la cogeneración y los aspectos referentes a su desarrollo resultan irrelevantes. Desde 1996 se identificaron los obstáculos a su desarrollo, y tienen que ver con la falta de acción gubernamental.

\section{Bibliografía}

ACEEE, "Federal tax strategies to encourage the adoption of combined heat and power", ACEEE Org., septiembre de 2003.

Cogen Europe, Who's Who in European Cogeneration 2000-2001, septiembre 2001.

CONAE, Elementos para la exposición de motivos y la elaboración de un régimen especial para la cogeneración en el mercado eléctrico mexicano. Mayo de 2002.

CRE, "Infocre", varios reportes, CRE, México 1998, 1999, 2000.

Energy Information Administration. Office of Coal, Nuclear, Electric and Alternate Fuels., Washington, DC, julio de 1998.

EPA Partnerships Division. "Market Development" EPA CHP Partners Meeting, Washington, DC, 2030 de abril de 2003.

http://info.main.conacyt.mx/damt/index.html http://info.main.conacyt.mx/damt/pmt/index.html, fondo de investigación y desarrollo para la modernización tecnológica.

http://rtn.net.mx.80/fidetec/

http://WWw.cipi.gob. $\mathrm{mx} / \mathrm{html} /$ programas_de_ apoyo.html, financiamientos directos, financiamientos indirectos, arancel cero para equipos anticontaminantes, créditos para el mejoramiento ambiental, créditos para infraestructura industrial. http://Www.nafin.gob.mx/, promin: programa único de financiamiento a la modernización industrial, programa de aportación accionaría, programa extraordinario de capital de riesgo, programa de desarrollo de proveedores, programa de apoyo a empresas integradoras, banco nacional de comercio exterior (BANCOMEXT).

de la CRE respecto al modo de otorgar permisos de cogeneración, promovida por el senador Manuel Barttlet desde la Comisión de Puntos Constitucionales en septiembre de 2003).

28 El método de objetivo y tarea obliga a que la administración especifique sus supuestos sobre la relación entre el capital empleado y los resultados de la promoción. Pero es también el método más difícil de utilizar. En efecto, a menudo resulta difícil definir qué tareas específicas servirán para alcanzar objetivos determinados. Con el citado método, la compañía establece un presupuesto basado en lo que quiere lograr con la promoción. 
León de los Santos, Gabriel, "Estudio de la viabilidad de la cogeneración industrial en México", tesis doctoral, DEPFI, UNAM, 2003.

Muñoz, José Manuel, “Cogeneración en grandes centrales de proceso", Unidad de innovación tecnológica, Subdirección técnica, CFE. 2000.

Nieva, Rolando, Hernández, Julio A., Portes, Enrique, Álvarez, José M., "Evaluación del potencial nacional de cogeneración como opción en la expansión del sistema eléctrico nacional". IIE, México, 1996. p. 19.

OCDE, Projected costs of generating electricity, Update 1998.

Rodríguez de Buen, Odón. "Estrategias de promoción de la cogeneración y generación de energía eléctrica a partir de energías renovables", Comisión Nacional Para el Ahorro de Energía, febrero de 1997.

Scott A., Spiewak/Larry Weiss, "Cogeneration \& small power production manual", Ed. Fairmont Press, Inc., 1994, p. 295.
Secretaría de Energía, Prospectiva del Sector Eléctrico 2003-2012, México, 2003.

Smith Merrill, "Department of Energy's CHP Program", Office of Distributed Energy and Electric Reability, U.S. Department of Energy. EPA CHP Partners Meeting, Washington, DC., 2030 de abrilde 2003.

Troche A., Luis, "Partnership Goals", EPA CHP Partners Meeting, Washington, DC, 20-30 de abril de 2003.

U.S. Department of Energy. "The Changing Structure of the Electric Power Industry". Selected Issues, 1998.

Van Der Does, Ton \& Brown, Michael, "The role of promotional organisations in the development of cogeneration", PW/K and Cogen Europe-1994. 